\title{
Modular Relative Jacobian for Combined 3-Arm Parallel Manipulators
}

\author{
Rodrigo S. Jamisola Jr. ${ }^{1}$, Carlos Mastalli ${ }^{2}$, and Frank Ibikunle ${ }^{1}$ \\ ${ }^{1}$ Electrical, Computer, and Telecommunications Engineering Dept., Botswana International University of Science and \\ Technology, Private Bag 16, Palapye, Botswana 10071 \\ ${ }^{2}$ Advanced Robotics Dept., Istituto Italiano di Tecnologia, Via Morego 30, 16163 Genova, Italy \\ Email: jamisolar@biust.ac.bw
}

\begin{abstract}
This work presents a new formulation of a modular relative Jacobian used to control combined manipulators as a single manipulator with a single effector. In particular, this modular relative Jacobian is designed for 3-arm parallel manipulators. It is called a relative Jacobian because it is expressed relative to the reference frames at the manipulator end-effectors. It is modular because it uses the existing information of each standalone manipulator component to arrive at the necessary expressions for the combined system. This work is part of a series of studies to express a single end-effector control of combined manipulators, in parallel as well as other types of base configurations. This holistic approach of controlling combined manipulators affords a drastic increase of the null-space dimension and the convenience to use all the principles of controlling a single manipulator for the resulting combined system. Derivation of the modular relative Jacobian for a 3-arm parallel manipulator is shown, together will simulation results.
\end{abstract}

Index Terms-modular kinematics, 3-arm parallel manipulators, single end-effector control, relative jacobian

\section{INTRODUCTION}

Modularity of manipulator kinematics and dynamics expressions have been recently actively studied because of the increasing complexity of robot structures. In particular, robots are no longer confined to single manipulators structures, but are now consisting of two or more manipulators combined together to form one single robot, like dual-arms, humanoids, quarupeds, hexapods, etc. Modular approach in the study of parallel robots has been used to many different types of applications, which include modular micro parallel robots [1], modular control architecture [2], modular design of parallel robots [3], kinematics and design of two variants [4], modular, wire-driven parallel robots [5], [6], design of modular parallel robots [7], multi-robot system ARGoS [8], and reconfigurable parallel robots [9], to name a few.

This study proposes to control combined 3-arm parallel manipulators (shown in Fig. 1) as a one single robot with a single end-effector. The advantage of this type of controller is two-fold: (1) it drastically increase the null-space dimension of the resulting combined

Manuscript received August 16, 2015; revised December 17, 2015. manipulators and (2) the principles of single manipulator control can now be applied to the combined manipulators For example, consider a dual-arm robot with each arm having six degrees-of-freedom (6-DOF). When each of the two arms is independently controlled in the full space, the resulting dual-arm robot is non-redundant. However, if the two arms are controlled in the relative full space, the resulting dual-arm robot has 6-DOF in the null space. In addition, through the single end-effector control, the combined manipulators can use a single manipulator controller, such that a strict task prioritization can now be implemented throughout the entire system.

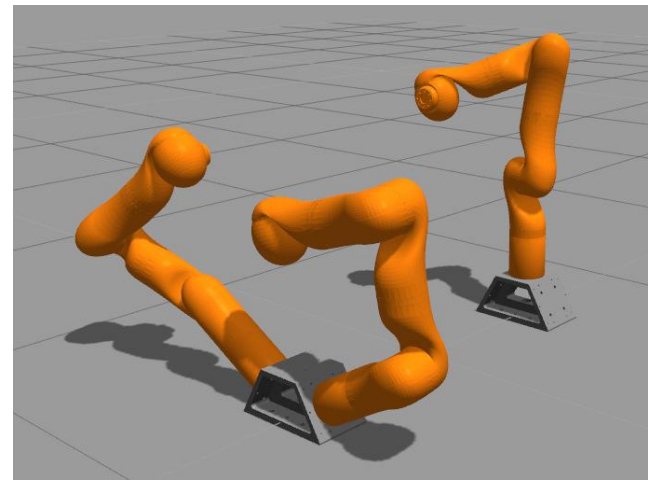

Figure 1. A 3-arm parallel manipulator. Simulation video is shown here: https://youtu.be/w87Ei7Z2Uis

Secondly, this study proposes modularity of the derived relative Jacobian. for the 3-arms. Modularity of the derived expression adds to the ease of implementation of the proposed single end-effector control. This is because the existing information of each of the standalone manipulator components will be used to arrive at the resulting expressions for the combined systems. Rotation and wrench transformation matrices are used to transform each of the standalone Jacobians to arrive at the relative Jacobian of the 3-arms.

A more compact modular relative Jacobian was first shown $\mathrm{n}$ [10], which reveals a wrench transformation matrix that was not present or was not explicitly expressed in the previous relative Jacobians. It was further shown that omission of the wrench transformation matrix can affect the performance of the dual-arm, including the forces and moments exerted at the endeffectors [11]. The concept of a relative Jacobian was 
first introduced in [12], [13]. A recent application of the relative Jacobian to asymmetric bimanual task was shown [14]. Other studies in robot kinematics include [15], [16].

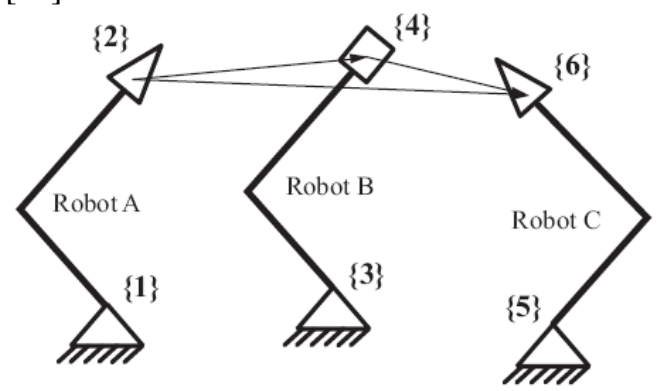

Figure 2. An schematic diagram of a 3-arm parallel manipulator, with the corresponding reference frames and the relative position vectors.

\section{NAMING CONVENTION FOR SYMBOLS}

In Fig. 2, the schematic diagram of a 3-arms parallel manipulator is shown, together with the corresponding reference frames. The base reference frames are oddnumbered, while the end-effector reference frames are even-numbered. The relative position vectors are also shown.

Consider reference frames $\{i\}$ and $\{j\}$, such that ${ }^{i} p_{j}$ is the position of frame $\{j\}$ with respect to frame $\{i\}$, and ${ }^{i} p_{j}$ is the rotation of frame $\{j\}$ with respect to frame $\{i\}$. In addition, a Jacobian ${ }^{i} J_{j}$ can be expressed with respect to those frames. From the figure, we state the following conventions for the Jacobians of the standalone manipulators. The Jacobian for robot $A$ is ${ }^{1} J_{2}$, for robot $B$ is ${ }^{3} J_{4}$, and for robot $C$ is ${ }^{5} J_{6}$, each is expressed with respect to the indicated reference frame indices.

\section{TABLE I. SYMBOLS-NAMING CONVENTION}

\begin{tabular}{ll} 
Sym. & Description \\
\hline${ }^{i} p_{j}$ & position of frame $\{j\}$ w.r.t. frame $\{i\} ;$ its first derivative is ${ }^{i} \dot{p}_{j}$ \\
${ }^{i} R_{j}$ & orientation of $\{j\}$ w.r.t. $\{i\}$; its first derivative is ${ }^{i} \dot{R}_{j}$ \\
${ }^{i} \omega_{j}$ & rotational velocity of $\{j\}$ w.r.t. $\{i\}$ \\
${ }^{i} J_{j}$ & {$\left[{ }^{i} J_{p j},{ }^{i} J_{o j}\right]^{T}$ Jacobian from $\{i\}$ to $\{j\}$} \\
${ }^{i} J_{p j}$ & position component of Jacobian ${ }^{i} J_{j}$ \\
${ }^{i} J_{o j}$ & orientation component of Jacobian ${ }^{i} J_{j}$ \\
${ }^{1} J_{2}$ & Jacobian of robot $A$ \\
${ }^{3} J_{4}$ & Jacobian of robot $B$ \\
${ }^{5} J_{6}$ & Jacobian of robot $C$ \\
${ }^{2} J_{4}$ & relative Jacobian of dual-arm robots $A$ and $B$ \\
${ }^{4} J_{6}$ & relative Jacobian of dual-arm robots $B$ and $C$ \\
${ }^{2} J_{6}$ & relative Jacobian of dual-arm robots $A$ and $C$ \\
${ }_{3}^{2} J_{6}$ & relative Jacobian of 3 -arm robots $A, B$ and $C$ \\
$\dot{q}_{2}$ & joint velocities of robot $A$ \\
$\dot{q}_{4}$ & joint velocities of robot $B$ \\
$\dot{q}_{6}$ & joint velocities of robot $C$ \\
$\dot{q}_{24}$ & {$\left[\dot{q}_{2}, \dot{q}_{4}\right]^{T}$ joint velocities of dual-arm robots $A$ and $B$} \\
$\dot{q}_{46}$ & {$\left[\dot{q}_{4}, \dot{q}_{6}\right]^{T}$ joint velocities of dual-arm robots $B$ and $C$} \\
$\dot{q}_{246}$ & {$\left[\dot{q}_{2}, \dot{q}_{4}, \dot{q}_{4}\right]^{T}$ joint velocities of 3 -arm robots $A, B$, and $C$} \\
${ }_{3}^{2} p_{6}$ & 3 -arm relative position of $\{6\}$ w.r.t. $\{2\}$ \\
$3_{3}^{2} \dot{p}_{6}$ & 3 -arm relative translational velocity of $\{6\}$ w.r.t. $\{2\}$ \\
${ }_{3}^{2} \omega_{6}$ & 3 -arm relative rotational velocity of $\{6\}$ w.r.t. $\{2\}$ \\
\hline
\end{tabular}

We assign the position Jacobian ${ }^{i} J_{p j}$ and orientation Jacobian ${ }^{i} J_{o j}$ as components of the Jacobian ${ }^{i} J_{j}$, that is, ${ }^{i} J_{j}=\left[{ }^{i} J_{p j},{ }^{i} J_{o j}\right]^{T}$. The joint velocities $\dot{q}_{i j}=\left[\dot{q}_{i}, \dot{q}_{j}\right]^{T}$, such that, $q_{i}$ and $q_{j}$ are the joint velocities of the robot with end-effector frames $\{i\}$ and $\{j\}$, respectively. For example, ${ }^{1} J_{2}=\left[{ }^{1} J_{p 2},{ }^{1} J_{o 2}\right]^{T}$ is the Jacobian for robot $A$, and ${ }^{2} J_{4}=\left[{ }^{2} J_{p 4},{ }^{2} J_{o 4}\right]^{T}$ is the relative Jacobian for robots $A$ and $B$. The dual-arm joint velocities $\dot{q}_{24}=\left[\dot{q}_{2}, \dot{q}_{4}\right]^{T}$, where $\dot{q}_{2}$ are the joint velocities of robot $A$ and $\dot{q}_{4}$ are the joint velocities of robot $B$. Naming convention for symbols and most symbols used in this work are shown in Table I.

\section{DERIVATION OF THE MODUlaR 3-ARM RELATIVE JACOBIAN}

In this section, we present the derivation of the modular relative Jacobian for three parallel manipulators. We derive the modular relative Jacobian of the 3-arm parallel manipulator by expressing the end-effector of the robot $C$ with respect to the end-effector of robot $A$. We will do this by taking two robots at a time, the same method that was invoked for the modular relative Jacobian of a dual-arm as derived in [10]. We show here the modular relative Jacobian for dual-arms as shown in [10], such that the relative Jacobian for a dual-arm consisting of robots $A$ and $B$ in Fig. 2 is

$$
{ }^{2} J_{4}=\left[\begin{array}{ll}
-{ }^{2} \Psi_{4}{ }^{2} \Omega_{1}{ }^{1} J_{2} & { }^{2} \Omega_{3}{ }^{3} J_{4}
\end{array}\right]
$$

and the relative Jacobian of a dual-arm consisting of robots $B$ and $C$ in Fig. 2 is

$$
{ }^{4} J_{6}=\left[-{ }^{4} \Psi_{6}{ }^{4} \Omega_{3}{ }^{3} J_{4} \quad{ }^{4} \Omega_{5}{ }^{5} J_{6}\right]
$$

Lastly, the relative Jacobian for dual-arm robots A and $\mathrm{C}$ is

$$
{ }^{2} J_{6}=\left[\begin{array}{ll}
-{ }^{2} \Psi_{6}{ }^{2} \Omega_{1}{ }^{1} J_{2} & { }^{2} \Omega_{5}{ }^{5} J_{6}
\end{array}\right]
$$

Such that the wrench transformation matrix ${ }^{i} \Psi_{j}$ is defined as

$$
{ }^{i} \Psi_{j}=\left[\begin{array}{cc}
I & -S\left({ }^{i} p_{j}\right) \\
0 & I
\end{array}\right]
$$

and the rotation matrix ${ }^{i} \Omega_{j}$ is expressed as

$$
{ }^{i} \Omega_{j}=\left[\begin{array}{cc}
{ }^{i} R_{j} & 0 \\
0 & { }^{i} R_{j}
\end{array}\right]
$$

Given $\omega=\left[\omega_{x}, \omega_{y}, \omega_{z}\right]^{T}$, the operator $S(\omega)$ is the skew symmetric operator used to replace the crossproduct operator and Jacobians is expressed as

$$
S(\omega)=\left[\begin{array}{ccc}
0 & -\omega_{z} & \omega_{y} \\
\omega_{z} & 0 & -\omega_{x} \\
-\omega_{y} & \omega_{x} & 0
\end{array}\right]
$$

To complete the definition of the modular dual-arm manipulators the shown robots in Fig. 2, we define the relative position vectors between the end-effectors, called ${ }^{i} p_{j}$ for the paired

$$
\begin{aligned}
{ }^{2} p_{4} & ={ }^{2} R_{1}\left({ }^{1} p_{3}+{ }^{1} R_{3}{ }^{3} p_{4}-{ }^{1} p_{2}\right) \\
{ }^{4} p_{6} & ={ }^{4} R_{3}\left({ }^{3} p_{5}+{ }^{3} R_{5}{ }^{5} p_{6}-{ }^{3} p_{4}\right) \\
{ }^{2} p_{6} & ={ }^{2} R_{1}\left({ }^{1} p_{5}+{ }^{1} R_{5}{ }^{5} p_{6}-{ }^{1} p_{2}\right) .
\end{aligned}
$$


Now we are ready to derive the modular relative Jacobian for the 3 -arm parallel manipulator, ${ }_{3}^{2} J_{6}$, by invoking a similar approach used in [10]. That is, we express translational and rotational velocities of the endeffectors with respect to each other. Thus the relative position of frame $\{6\}$ with respect to frame $\{2\}$ can be expressed as

$$
{ }_{3}^{2} p_{6}={ }^{2} p_{4}+{ }^{2} R_{4}{ }^{4} p_{6}
$$

and taking the derivative of the above equation results in

$$
\begin{aligned}
{ }_{3}^{2} \dot{p}_{6} & ={ }^{2} \dot{p}_{4}+{ }^{2} \dot{R}_{4}{ }^{4} p_{6}+{ }^{2} R_{4}{ }^{4} \dot{p}_{6} \\
& ={ }^{2} \dot{p}_{4}+S\left({ }^{2} \omega_{4}\right)^{2} R_{4}{ }^{4} p_{6}+{ }^{2} R_{4}{ }^{4} \dot{p}_{6} \\
& ={ }^{2} \dot{p}_{4}-S\left({ }^{2} R_{4}{ }^{4} p_{6}\right)^{2} \omega_{4}+{ }^{2} R_{4}{ }^{4} \dot{p}_{6}
\end{aligned}
$$

Because angular velocities are linear, we can express the relative angular velocity of frame $\{6\}$ with respect to frame $\{2\}$ as

$$
{ }_{3}^{2} \omega_{6}={ }^{2} \omega_{4}+{ }^{2} R_{4}{ }^{4} \omega_{6}
$$

Combining (9) and (10) we get

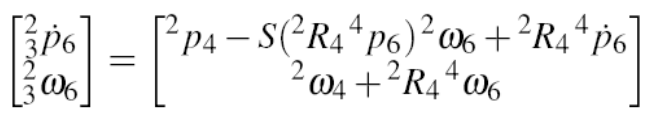

We express the above expression in terms of the relative Jacobians

$$
\begin{aligned}
& {\left[\begin{array}{ll}
2 & \dot{p}_{6} \\
2 & \omega_{3}
\end{array}\right]} \\
& =\left[\begin{array}{c}
{ }^{2} J_{p 4} \dot{q}_{24}-S\left({ }^{2} R_{4}{ }^{4} p_{6}\right){ }^{2} J_{o 4} \dot{q}_{24}+{ }^{2} R_{4}{ }^{4} J_{p 6} \dot{q}_{46} \\
{ }^{2} J_{o 4} \dot{q}_{24}+{ }^{2} R_{4}{ }^{4} J_{o 6} \dot{q}_{46}
\end{array}\right] \\
& =\left[\left[\begin{array}{cc}
I & -S\left({ }^{2} R_{4}{ }^{4} p_{6}\right) \\
0 & I
\end{array}\right]\left[\begin{array}{c}
{ }^{2} J_{p 4} \\
{ }^{2} J_{o 4}
\end{array}\right] \dot{q}_{24} \ldots\right. \\
& \left.\ldots+\left[\begin{array}{cc}
{ }^{2} R_{4} & 0 \\
0 & { }^{2} R_{4}
\end{array}\right]\left[\begin{array}{c}
{ }^{4} J_{p 6} \\
{ }^{4} J_{o 6}
\end{array}\right] \dot{q}_{46}\right] \\
& \left.=\left[\begin{array}{cc}
I & -S\left({ }^{2} R_{4}{ }^{4} p_{6}\right) \\
0 & I
\end{array}\right]{ }^{2} J_{4} \quad\left[\begin{array}{cc}
{ }^{2} R_{4} & 0 \\
0 & { }^{2} R_{4}
\end{array}\right]{ }^{4} J_{6}\right]\left[\begin{array}{c}
\dot{q}_{24} \\
\dot{q}_{46}
\end{array}\right] \\
& =\left[\begin{array}{lll}
2,4 & \Psi_{6}{ }^{2} J_{4} & { }^{2} \Omega_{4}{ }^{4} J_{6}
\end{array}\right]\left[\begin{array}{l}
\dot{q}_{24} \\
\dot{q}_{46}
\end{array}\right] \\
& =\left[{ }^{2,4} \Psi_{6}\left[-{ }^{2} \Psi_{4}{ }^{2} \Omega_{1}{ }^{1} J_{2}{ }^{2} \Omega_{3}{ }^{3} J_{4}\right] \ldots\right. \\
& \left.\ldots{ }^{2} \Omega_{4}\left[\begin{array}{lll}
-{ }^{4} \Psi_{6}{ }^{4} \Omega_{3}{ }^{3} J_{4} & { }^{4} \Omega_{5}{ }^{5} J_{6}
\end{array}\right]\right]\left[\begin{array}{l}
\dot{q}_{24} \\
\dot{q}_{46}
\end{array}\right] \\
& =\left[-{ }^{2,4} \Psi_{6}{ }^{2} \Psi_{4}{ }^{2} \Omega_{1}{ }^{1} J_{2} \quad\left({ }^{2,4} \Psi_{6}{ }^{2} \Omega_{3}-{ }^{2} \Omega_{4}{ }^{4} \Psi_{6}{ }^{4} \Omega_{3}\right)^{3} J_{4} \ldots\right. \\
& \left.\ldots{ }^{2} \Omega_{4}{ }^{4} \Omega_{5}{ }^{5} J_{6}\right]\left[\begin{array}{l}
\dot{q}_{24} \\
\dot{q}_{46}
\end{array}\right]
\end{aligned}
$$

In the second to the last equality of (12), we substitute the dual-arm relative Jacobians of (1) and (2). To further simplify, we group terms together, such that the modular relative Jacobian for a 3-arm parallel manipulator can be expressed as

$$
\begin{array}{r}
{ }_{3}^{2} J_{6} \\
=\left[\begin{array}{lr}
-{ }^{2,4} \Psi_{6}{ }^{2} \Psi_{4}{ }^{2} \Omega_{1}{ }^{1} J_{2} & \left({ }^{2,4} \Psi_{6}{ }^{2} \Omega_{3}-{ }^{2} \Omega_{4}{ }^{4} \Psi_{6}{ }^{4} \Omega_{3}\right){ }^{3} J_{4} \ldots \\
\left.\ldots{ }^{2} \Omega_{4}{ }^{4} \Omega_{5}{ }^{5} J_{6}\right]
\end{array}\right.
\end{array}
$$

where ${ }^{i, j} \Psi_{k}$ means that the wrench transformation matrix has the cross-product operator defined as $S\left({ }^{i} R_{j}{ }^{j} p_{k}\right)$.
We then need to simplify (13) column by column. We invoke Matlab matrix notation to do this. Thus the first column of ${ }_{3}^{2} J_{6}$ is

$$
\begin{aligned}
{ }_{3}^{2} J_{6}(:, 1) & =-{ }^{2,4} \Psi_{6}{ }^{2} \Psi_{4}{ }^{2} \Omega_{1}{ }^{1} J_{2} \\
& =-\left[\begin{array}{cc}
I & -S\left({ }^{2} R_{4}{ }^{4} p_{6}\right) \\
0 & I
\end{array}\right]\left[\begin{array}{cc}
I & -S\left({ }^{2} p_{4}\right) \\
0 & I
\end{array}\right]{ }^{2} \Omega_{1}{ }^{1} J_{2} \\
& =-\left[\begin{array}{cc}
I & -S\left({ }^{2} p_{4}+{ }^{2} R_{4}{ }^{4} p_{6}\right) \\
0 & I
\end{array}\right]{ }^{2} \Omega_{1}{ }^{1} J_{2} \\
& =-\left[\begin{array}{cc}
I & -S\left({ }^{2} p_{6}\right) \\
0 & I
\end{array}\right]{ }^{2} \Omega_{1}{ }^{1} J_{2} \\
& =-{ }^{2} \Psi_{6}{ }^{2} \Omega_{1}{ }^{1} J_{2} .
\end{aligned}
$$

The second column of ${ }_{3}^{2} J_{6}$ is

$$
\begin{aligned}
& { }_{3}^{2} J_{6}(:, 2) \\
& =\left({ }^{2,4} \Psi_{6}{ }^{2} \Omega_{3}-{ }^{2} \Omega_{4}{ }^{4} \Psi_{6}{ }^{4} \Omega_{3}\right){ }^{3} J_{4} \\
& =\left({ }^{2,4} \Psi_{6}{ }^{2} \Omega_{3}-\left[\begin{array}{cc}
{ }^{2} R_{4} & 0 \\
0 & { }^{2} R_{4}
\end{array}\right]\left[\begin{array}{cc}
I & -S\left({ }^{4} p 6\right) \\
0 & I
\end{array}\right]{ }^{4} \Omega_{3}\right){ }^{3} J_{4} \\
& =\left({ }^{2,4} \Psi_{6}{ }^{2} \Omega_{3}-\left[\begin{array}{cc}
{ }^{2} R_{4} & -{ }^{2} R_{4} S\left({ }^{4} p_{6}\right)^{2} R_{4}^{T}{ }^{2} R_{4} \\
0 & { }^{2} R_{4}
\end{array}\right]{ }^{4} \Omega_{3}\right){ }^{3} J_{4} \\
& =\left({ }^{2,4} \Psi_{6}{ }^{2} \Omega_{3}-\left[\begin{array}{cc}
{ }^{2} R_{4} & -S\left({ }^{2} R_{4}{ }^{4} p_{6}\right){ }^{2} R_{4} \\
0 & { }^{2} R_{4}
\end{array}\right]{ }^{4} \Omega_{3}\right){ }^{3} J_{4} \\
& =\left({ }^{2,4} \Psi_{6}{ }^{2} \Omega_{3}-\left[\begin{array}{cc}
I & -S\left({ }^{2} R_{4}{ }^{4} p_{6}\right) \\
0 & I
\end{array}\right]\left[\begin{array}{cc}
{ }^{2} R_{4} & 0 \\
0 & { }^{2} R_{4}
\end{array}\right]{ }^{4} \Omega_{3}\right){ }^{3} J_{4} \\
& =\left({ }^{2,4} \Psi_{6}{ }^{2} \Omega_{3}-{ }^{2,4} \Psi_{6}{ }^{2} \Omega_{3}\right){ }^{3} J_{4} \\
& =0 \text {. }
\end{aligned}
$$

This make the relative Jacobian of the 3-arm parallel manipulator to be

$$
{ }_{3}^{2} J_{6}=\left[-{ }^{2} \Psi_{6}{ }^{2} \Omega_{1}{ }^{1} J_{2} \quad 0 \quad{ }^{2} \Omega_{5}{ }^{5} J_{6}\right]
$$

which is identical to (3), except for the middle zero column.

Thus, in this type of formulation, the third arm will always move in the null-space of the dual arm. A holistic modular kinematic expression for the 3-arm parallel manipulator can be expressed as

$$
\begin{array}{r}
\dot{q}_{246}={ }_{3}^{2} J_{6}^{+2} x_{6}+\left(I-{ }_{3}^{2} J_{6}^{+2}{ }_{3}^{2} J_{6}\right)_{3}^{2} J_{4}^{+2} x_{4} \ldots \\
\ldots+\left(I-{ }_{3}^{2} J_{6}^{+2}{ }_{3} J_{6}\right)\left(I-{ }_{3}^{2} J_{4}^{+2}{ }_{3}^{2} J_{4}\right) \nabla z
\end{array}
$$


and $\nabla z$ is the null space posture. The null space projection of $\nabla z$ can be computed as shown in [17], where maximum number of tasks was utilized and prioritized despite singularities. The expression in (17) shows that modularity of the kinematics expressions for null space is achieved in both end-effector and null-space motions.

\section{SimUlation Using GAZEBO}

This section presents simulation results of a 3-arm parallel manipulator using the modular relative Jacobian derived in the previous section. Robot simulator Gazebo 2.2.5 is used as the simulation platform with Robot Operating Systems (ROS) Indigo. A Universal Robotic Description Format (URDF) of the 7-DOF KUKA LWR was created. The simulation is running under Ubuntu 
Trusty 14.04 LTS 64-bit with Intel Corei5-4210U quadcore processor.

\section{A. Simulation Controller}

The controller in the simulation is a controller with purely kinematic information, without any dynamics information included. This can be a limitation in the simulation. The simulator takes in torque inputs from the controller. And because the implemented control is purely a kinematics controller, the output of the velocity controller stated below was directly converted to torques and passed to the simulator. The velocity controller is expressed as

$$
\begin{aligned}
\dot{q}_{246} & =J_{R}^{+} \Delta\left(x_{R}\right)+\left(I-J_{R}^{+} J_{R}\right){ }_{3}^{1} J_{2}^{+} \Delta\left({ }^{1} x_{2}\right) \\
\ldots+ & \left(I-J_{R}^{+} J_{R}\right)\left(I-{ }_{3}^{1} J_{2}^{+}{ }_{3} J_{2}\right){ }_{3}^{2} J_{4}^{+} \Delta\left({ }^{2} x_{4}\right) \ldots \\
\ldots+ & \left(I-J_{R}^{+} J_{R}\right)\left(I-{ }_{3}^{1} J_{2}^{+}{ }_{3} J_{2}\right)\left(I-{ }_{3}^{2} J_{4}^{+2} J_{3}\right) \nabla z
\end{aligned}
$$

where $J_{R}=\frac{2}{3} J_{6}$ and $x_{R}={ }_{3}^{2} x_{6}$ is the relative position and orientation vector. For the delta function, given $\mathrm{x}$ as the input,

$$
\Delta(x)=k_{P}\left(x_{d}-x\right)+k_{V}\left(\dot{x}_{d}-\dot{x}\right)+k_{I} \sum_{t=0}\left(x_{d}-x\right)
$$

where $x_{d}$ is the desired $x, \dot{x}_{d}$ desired velocity of $x_{d}, \dot{x}$ is the velocity of $x, t$ is the time, and $k_{p}, k_{v}$, and $k_{i}$ are the proportional, derivative, and integral gains. The $3-$ arm null-space Jacobians are ${ }_{3}^{2} J_{4}=\left[\begin{array}{ll}{ }^{2} J_{4} & 0\end{array}\right]$ and ${ }_{3}^{1} J_{2}=\left[\begin{array}{lll}{ }^{1} J_{2} & 0 & 0\end{array}\right]$. The $\nabla z$ is the null-space gradient that controls the posture of the arms, such that $\nabla(z)=\left[\Delta\left(q_{2}\right), \Delta\left(q_{4}\right), \Delta\left(q_{6}\right)\right]^{T}$.

For (18), the first term on the left hand side of the equation controls the relative motion of robot $C$ endeffector with respect to the robot $A$ end-effector, in a dual-arm kind of control strategy. In this approach, the end-effector of robot $B$ lies in the null space. The second term controls the relative motion of robot $\mathrm{B}$ end-effector with respect to robot $A$ end-effector. The third term moves the robot $A$ end-effector (which is the overall reference frame) with respect to the world frame. Because the end-effectors of robot $B$ and $C$ moves with respect to the robot $A$ end-effector, all three end-effectors will move, as robot $A$ end-effector is moving. The desired robot posture as defined in $\nabla z$ is accommodated as long as it does not have any conflict with the three other higher priority tasks.

\section{B. The Desired Values}

The desired values are the following (with lengths in meters and angles in degrees): ${ }_{3}^{2} x_{6 d}=[0,0,0.3,0,180,0]^{T}$ ( $x, y$, and $z$ position and roll, pitch and yaw orientation), ${ }^{2} x_{4 d}=[-0.3,0,0,0,0,0]^{T}, \quad q_{2 d}=[0,+60,0,-45,0,-45,0]^{T}$, $q_{4 d}=[0,-60,0,+45,0,+45,0]^{T}: \quad$ and $\quad q_{6 d}:=$ $[0,+60,0,-45,0,-45,0]^{T}$. All desired velocities are zero. The desired values ${ }^{1} x_{2 d}$ changes according in a pointto-point motion to the time increment of $1 \mathrm{~s}$ as follows: $\quad{ }^{1} x_{2 d}=[0.5,0,0.5,90,0,0]_{t=0}^{T}$, ${ }^{1} x_{2 d}=[0.5,-0.5,0.5,90,0,0]_{t=1}^{T},{ }_{-}^{1} x_{2 d}=[0,-0.5,0.5,90,0,0]_{t=2}^{T}$, and ${ }^{1} x_{2 d}=[0,0,0.5,90,0,0]_{t=3}^{T}$. Then ${ }^{1} x_{2 d}$ loops back in a $4 s$ cycle of desired values. $A$ simulation of the described desired motion is shown in Fig. 3.

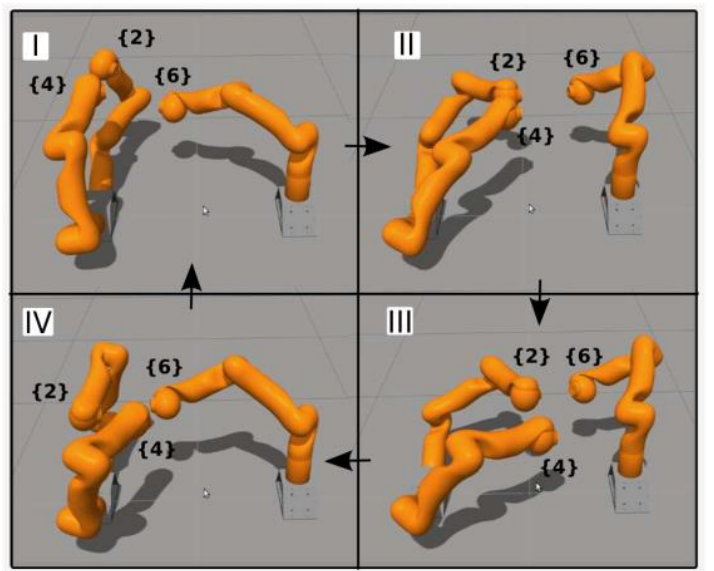

Figure 3. Snapshots of Gazebo simulation where the 3-arm parallel manipula- tors move in a coordinated manner, in single manipulator control.

Note that Gazebo simulator does not run in real-time. The gains are set at $k_{P}(1: 3)=3000$ for position and $k_{P}(4: 6)=1500$ for orientation, $k_{V}=200$, and $k_{I}=0.1$. Note that the $\Delta(q)$ function in the null-space used $k_{P}=200$, and $k_{V}=k_{I}=0$. Now we are ready to show the simulation results.

\section{Numerical Results}

The error results of the numerical simulation from the Gazebo simulation in Fig. 3 are shown from Figs. 4 to 10. The end-effector of robot $A$ (frame $\{2\}$ ) is the reference frame for the motion of robot $\mathrm{C}$ end-effector (frame $\{6\}$ ) and motion of robot $B$ end-effector (frame $\{4\}$ ). However, motion of $\{6\}$ with respect to $\{2\}$ is the highest priority, as shown in (18). Second priority is the motion of $\{2\}$ with respect to its base, third priority is the motion of $\{4\}$ with respect to $\{2\}$, and last priority is the null-space posture of the joints

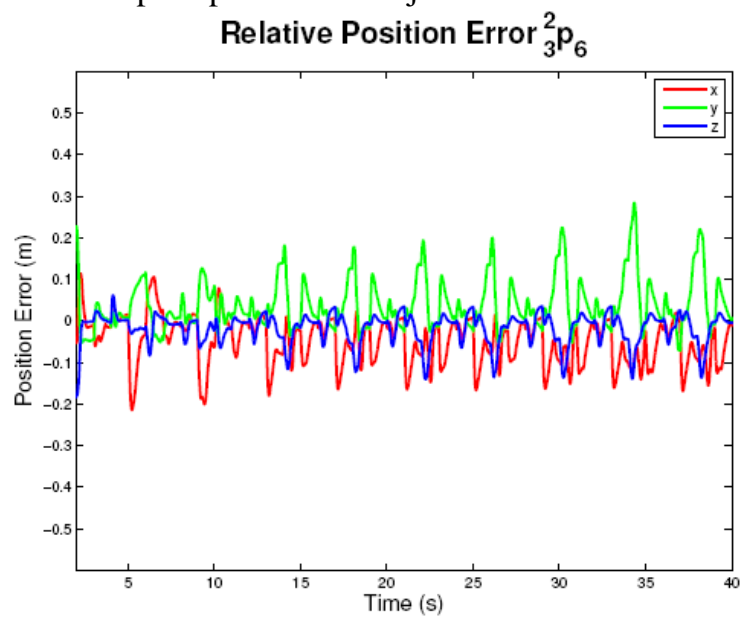

Figure 4. The relative position error ${ }_{3}^{2} p_{6}$

For the entire motion, only $\{2\}$ is controlled to move at an unending square path while the relative position and orientation of $\{6\}$ and $\{4\}$ with respect to $\{2\}$ is 
fixed at the desired values. A video of the experiment is shown here: https://youtu.be/w87Ei7Z2Uis. The resulting motion is that all the end-effectors are moving as a result of specified relative motion, according to the hierarchy of task prioritization of a single manipulator control.

Relative Orientation Error ${ }_{3}^{2} \omega_{6}$



Figure 5. The relative orientation $\operatorname{error}_{3}^{2} \omega_{6}$

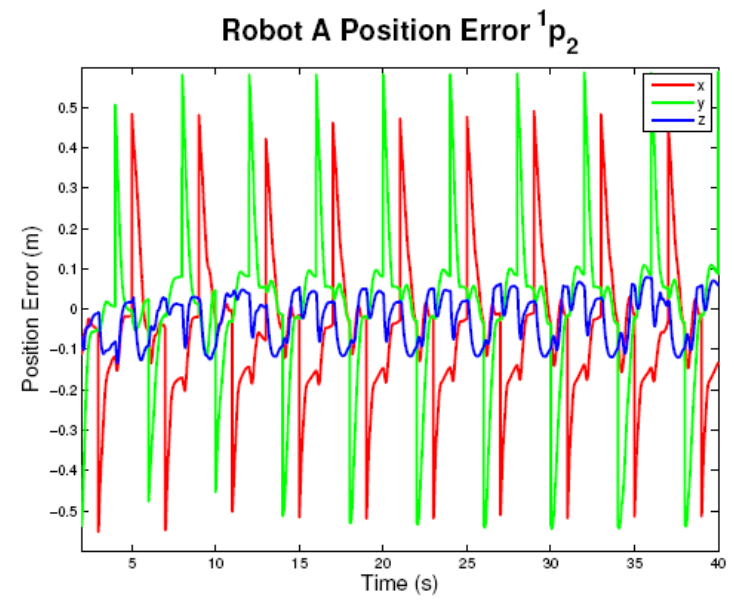

Figure 6. The robot $A$ position error ${ }^{1} p_{2}$

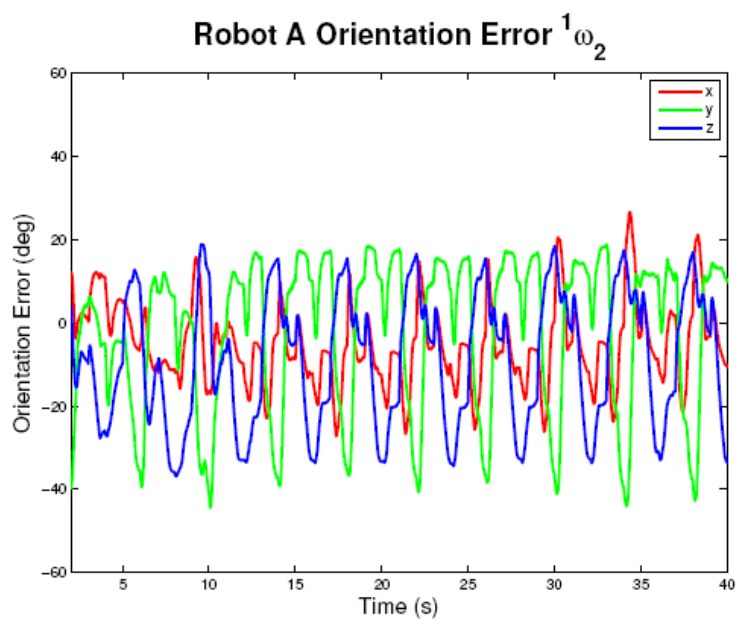

Figure 7. The robot $A$ orientation error ${ }^{1} \omega_{2}$

Thus, the least position error is reflected by the error in ${ }_{3}^{2} p_{6}$ shown in Fig. 4, the task with the highest priority. In most cases, the task with the higher priority has the least errors compared the less priority tasks, except when at certain manipulator configurations that are difficult to achieve for the given desired values. The results of this simulation can be greatly improved when the dynamics of the system is compensated or canceled in the controller.

Relative Position Error ${ }^{2} \mathrm{p}_{4}$

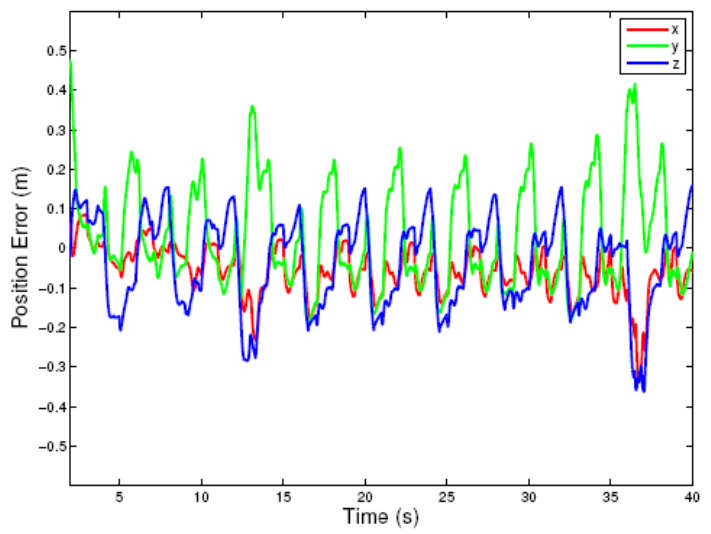

Figure 8 . The relative position error ${ }^{2} p_{4}$

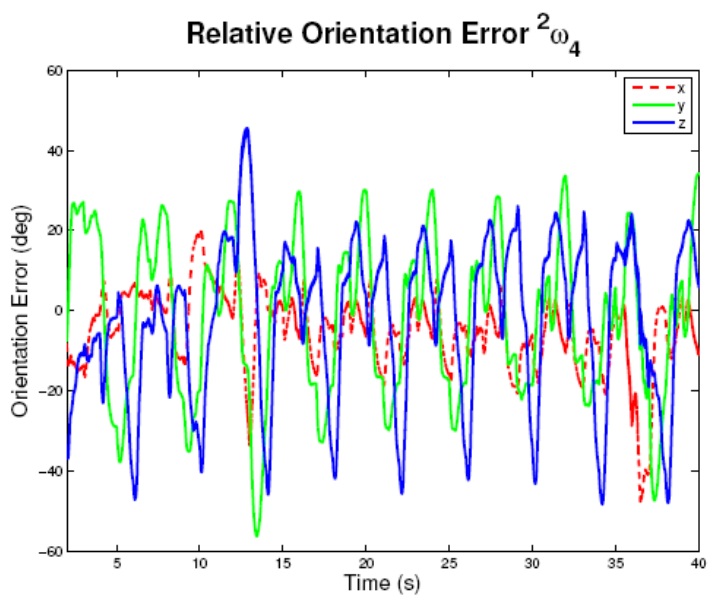

Figure 9. The relative position error ${ }^{2} \omega_{4}$

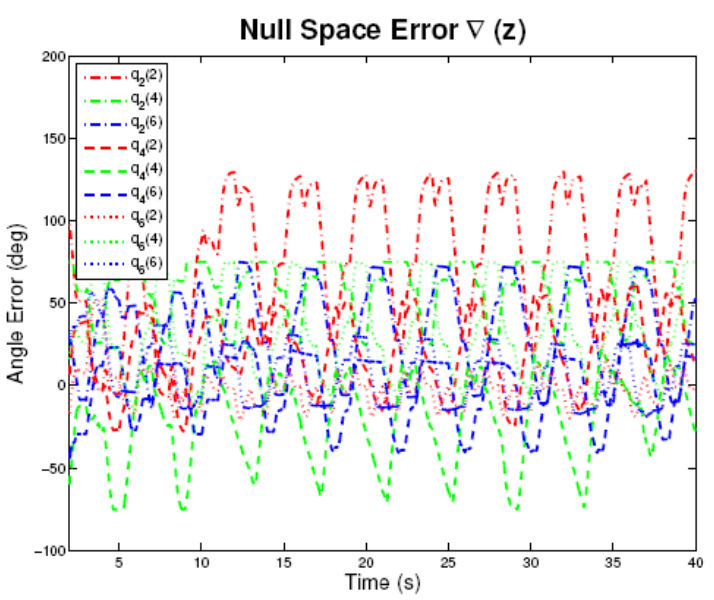

Figure 10. The null space posture error $\nabla z$

\section{CONCLUSION}

This work derived a modular relative Jacobian of a 3arm parallel manipulator, based on the dual-arm relative 
Jacobian approach of computation. In this new expression, the Jacobian of the third manipulator always lie in the null of the overall Jacobian. In addition, it was shown that this approach affords a task prioritization control that is effectively a single manipulator, and thus task prioritization can be strictly implemented.

\section{REFERENCES}

[1] C. Ng, S. Ong, and A. Y. Nee, "Design and development of 3dof modular micro parallel kinematic manipulator," The International Journal of Advanced Manufacturing Technology, vol. 31, no. 1-2, pp. 188-200, 2006.

[2] J. Maaß, N. Kohn, and J. Hesselbach, "Open modular robot control architecture for assembly using the task frame formalism," International Journal of Advanced Robotic Systems, vol. 3, no. 1, pp. 1-10, 2006

[3] A. E. Graham, S. Q. Xie, K. C. Aw, W. Xu, and S. Mukherjee, "Design of a parallel long bone fracture reduction robot with planning treatment tool," in Proc. IEEE/RSJ International Conference on Intelligent Robots and Systems, 2006, pp. 12551260.

[4] D. Pisla, N. Plitea, A. Vidrean, B. Prodan, B. Gherman, and D. Lese, "Kinematics and design of two variants of a reconfigurable parallel robot," in Proc. ASME/IFToMM International Conference on Reconfigurable Mechanisms and Robots, 2009, pp. 624-631.

[5] J. P. Merlet, "Marionet, a family of modular wire-driven parallel robots," in Advances in Robot Kinematics: Motion in Man and Machine, Springer, 2010, pp. 53-61.

[6] J. P. Merlet and D. Daney, "A portable, modular parallel wire crane for rescue operations," in Proc. International Conference on Robotics and Automation, 2010, pp. 2834-2839.

[7] C. E. Syrseloudis, I. Z. Emiris, T. Lilas, and A. Maglara, "Design of a simple and modular 2-dof ankle physiotherapy device relying on a hybrid serial-parallel robotic architecture," Applied Bionics and Biomechanics, vol. 8, no. 1, pp. 101-114, 2011.

[8] C. Pinciroli, V. Trianni, R. OGrady, G. Pini, A. Brutschy, M. Brambilla, et al., "Argos: Modular, parallel, multi-engine simulator for multi-robot systems," Swarm Intelligence, vol. 6, no. 4, pp. 271-295, 2012.

[9] G. J. Hamlin and A. C. Sanderson, "Tetrobot: A modular approach to reconfigurable parallel robotics," Springer Science \& Business Media, 2013, vol. 423.

[10] R. S. Jamisola and R. G. Roberts, "A more compact expression of relative jacobian based on individual manipulator jacobians," Robotics and Autonomous Systems, vol. 63, pp. 158-164, 2015.

[11] R. S. Jamisola Jr, P. Kormushev, D. G. Caldwell, and F. Ibikunle, "Modular relative jacobian for dual-arms and the wrench transformation matrix," in Proc. 7th IEEE International Conference on Cybernetics and Intelligent Systems (CIS) Robotics, Automation and Mechatronics (RAM), At Angkor Wat, Cambodia, 2015.

[12] C. Lewis and A. Maciejewski, "Trajectory generation for cooperating robots," in Proc. IEEE International Conference on Systems Engineering, 1990, pp. 300-303.

[13] C. Lewis, "Trajectory generation for two robots cooperating to perform a task" in Proc. IEEE International Conference on Robotics and Automation, Apr. 1996, vol. 2, pp. 1626-1631.

[14] J. Lee, P. Chang, and R. S. Jamisola, "Relative impedance control for dual-arm robots performing asymmetric bimanual tasks," IEEE Transactions on Industrial Electronics, vol. 61, no. 7, pp. 3786-3796, 2014

[15] R. S. Jamisola, A. A. Maciejewski, and R. G. Roberts, "Failuretolerant path planning for kinematically redundant manipulators anticipating locked-joint failures," IEEE Transactions on Robotics, vol. 22, no. 4, pp. 603-612, 2006.
[16] R. Jamisola, "Optimization of failure-tolerant workspaces for redundant manipulators," Philippine Science Letters., pp. 66-75, 2010.

[17] R. S. Jamisola, P. H. Chang, and J. Lee, "Guaranteeing task prioritization for redundant robots given maximum number of tasks and singularities," in Proc. TENCON IEEE Region 10 Conference, 2012, pp. 1-6.

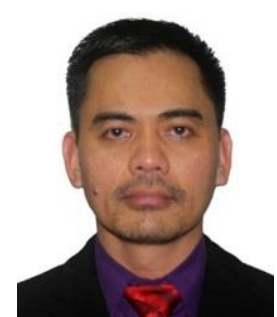

Rodrigo S. Jamisola, Jr. received his B.S. degree in mechanical engineering from the University of the Philippines-Diliman, M.E. degree (research-based) in mechanical engineering from the National Uni- versity of Singapore in 2001, M.Sc. degree in electrical and computer engineering from Colorado State University in 2006, and Ph.D. degree in electronics and communications engineering from De La Salle UniversityManila in 2009. He joined De La Salle University as an Asst. Professor in 2008 and Toyota Motor Philippines as R\&D Manager in 2011. He was a Post-doctoral Research Fellow at Daegu-Gyeongbuk Institute of Science and Technology in South Korea, and then at Istituto Italiano di Tecnologia in Genova, Italy. He is currently a Sr. Lecturer at Botswana International University of Science and Technology. His research interest includes control of combined manipulators, machine learning, numerical optimization, and human-machine interfaces.

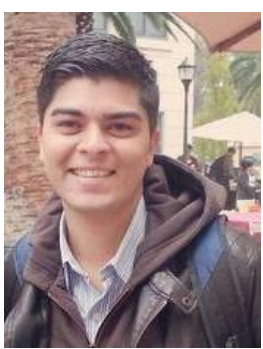

Carlos Mastalli received his Bachelor's Degree in Mechanical Engineering and Master's Degree in Mechatronic and Robotics from Antonio Jose de Sucre University and Simon Bolivar University, in 2008 and 2013 respectively. During 2011 to 2013, he was working as a Lecturer at Process and Systems Dept. of Simon Bolivar University and, at the same time, he was working as a Junior Researcher in the Mechatronic R\&D Group. In the past, he has worked in the development of Autonomous Backhoe Machines using Learning from Demonstration and an open-source library for Model Predictive Control. In 2014 he started as PhD-fellow in Dynamic Legged Systems Lab of Italian Institute of Tech-nology. Carlos Mastalli is interested in legged locomotion, motion planning, optimization and reinforcement learning techniques, robot learning, optimal control and perception systems

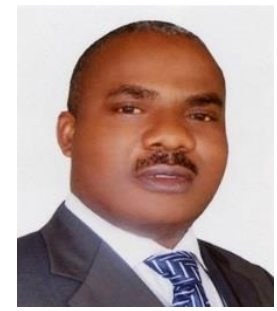

Frank Ayo Ibikunle is a Professor in Telecom- munications Engineering and the HoD of Electri- cal, Computer and Telecommunications Engineering Department at Botswana International University of Science and Technology (BIUST). He received his first degree in Electrical/Electronic Engineering and won a Scholarship award to study for his Masters and $\mathrm{PhD}$ degrees in Information and Telecommu- nications Engineering. He has 26 years of industrial/practical working experience in the Telecom- munications/ICT companies before joining the academic. He lectured at Covenant University in the Electrical \& Information Engineering Department, Nigeria before joining BIUST. His areas of research are in Mobile and Broadband Wireless Access Technologies (i.e. OFDMA, MIMO, Satellite, WiFi/WiMAX \& 2G/3G/LTE); Next Generation Converged Networks; Communication Network and Security; Cognitive radio, Adhoc and Sensor networks; Artificial Intelligence concepts (i.e., Neural Networks, Expert systems \& Fuzzy logics); Cloud Computing and its application; and Radio Channel Propagation \& Modelling in Wireless communications. 